\title{
Efficacy and safety of mecapegfilgrastim for prophylaxis of chemotherapy-induced neutropenia in patients with breast cancer: a randomized, multicenter, active-controlled phase III trial
}

\author{
Fengrui Xu ${ }^{1,2 \#}$, Yang Zhang ${ }^{3 \#}$, Zhanhui Miao ${ }^{4}$, Xiaohua Zeng ${ }^{5}$, Biao $\mathrm{Wu}^{6}$, Li $\mathrm{Cai}^{7}$, Jinping Liu ${ }^{8}$, \\ Shusen Wang ${ }^{9}$, Xichun $\mathrm{Hu}^{10}$, Wenbo Zheng ${ }^{11}$, Zhiyue Chen ${ }^{12}$, Qing Yang ${ }^{12}$, Zefei Jiang ${ }^{1}$ \\ ${ }^{1}$ Department of Breast Oncology, The Fifth Medical Center of Chinese PLA General Hospital, Beijing 100071, China; ${ }^{2}$ Department of Breast \\ Oncology, Academy of Military Medical Sciences, Beijing 100089, China; ${ }^{3}$ Department of Breast Surgery, Liaocheng People's Hospital, Liaocheng \\ 252000, China; ${ }^{4}$ Department of Medical Oncology, The First Affiliated Hospital of Xinxiang University, Xinxiang 453100, China; ${ }^{5}$ Department \\ of Breast Surgery, Chongqing Cancer Hospital, Chongqing 400030, China; ${ }^{6}$ Department of General Surgery, The First Affiliated Hospital of \\ Nanchang University, Nanchang 330006, China; ${ }^{7}$ Department of Medical Oncology, Harbin Medical University Cancer Hospital, Harbin 150081, \\ China; ${ }^{8}$ Department of Breast Surgery, Sichuan Province People's Hospital, Chengdu 610072, China; ${ }^{9}$ Department of Medical Oncology, Zhongshan \\ University Cancer Center, Guangzhou 510060, China; ${ }^{10}$ Department of Medical Oncology, Fudan University Shanghai Cancer Center, Shanghai \\ 200032, China; ${ }^{11}$ Department of Breast Surgery, The First Affiliated Hospital of Guangzhou Medical University, Guangzhou 510120, China; \\ ${ }^{12}$ Department of Research and Development, Jiangsu Hengrui Medicine Co., Ltd., Shanghai 200120, China \\ Contributions: (I) Conception and design: Z Jiang; (II) Administrative support: Z Jiang; (III) Provision of study materials or patients: All authors; \\ (IV) Collection and assembly of data: All authors; (V) Data analysis and interpretation: Z Jiang, F Xu, Z Chen, Q Yang; (VI) Manuscript writing: All \\ authors; (VII) Final approval of manuscript: All authors.
}

\#These authors contributed equally to this work.

Correspondence to: Zefei Jiang. Department of Breast Oncology, The Fifth Medical Center of Chinese PLA General Hospital, No. 8 East Street, Fengtai District, Beijing 100071, China. Email: jiangzefei@csco.org.cn.

Background: Neutropenia is a common complication from chemotherapy. Mecapegfilgramtim (code name HHPG-19K), a long-acting recombinant human granulocyte colony-stimulating factor (rhG-CSF), has been developed. This study was to evaluate the efficacy and safety of mecapegfilgrastim for reducing neutropenia compared with filgrastim.

Methods: This was a randomized, controlled non-inferiority study. A total of 339 breast cancer patients who were eligible for (neo) adjuvant chemotherapy were randomized assigned into three groups to receive mecapegfilgrastim $100 \mu \mathrm{g} / \mathrm{kg}$, mecapegfilgrastim fixed dose of $6 \mathrm{mg}$ or filgrastim $5 \mu \mathrm{g} / \mathrm{kg} / \mathrm{day}$ in the first cycle of chemotherapy. The primary endpoint was the duration of grade $\geq 3$ neutropenia in cycle 1 . The secondary endpoints included the duration of grade $\geq 3$ neutropenia in cycles $2-4$, incidence of grade $\geq 3$ neutropenia, and febrile neutropenia (FN). The safety profile was also evaluated.

Results: The mean duration of grade $\geq 3$ neutropenia was 1.06 [95\% confidence interval (CI): $0.65,1.26$ ] days in mecapegfilgrastim $100 \mu \mathrm{g} / \mathrm{kg}$ group, 1.23 (95\% CI: 0.84, 1.88) days in mecapegfilgrastim $6 \mathrm{mg}$ group, and 2.06 (95\% CI: 1.66, 2.46) days in the filgrastim group. The mean difference between mecapegfilgrastim $100 \mu \mathrm{g} / \mathrm{kg}$ and filgrastim was -1.00 (95\% CI: $-1.52,-0.48)$, the mean difference between mecapegfilgrastim $6 \mathrm{mg}$ and filgrastim was -0.83 (95\% CI: $-1.36,-0.30)$. The upper bounds of $95 \%$ CI for the difference between mecapegfilgrastim and filgrastim were all $<1$ day (the predefined non-inferiority margin). For the incidence of grade $\geq 3$ and grade 4 neutropenia, the mean duration of grade 4 neutropenia, mecapegfilgrastim showed better performance compared with filgrastim. For the incidence of FN, there was no difference between patients treated with mecapegfilgrastim and filgrastim. For safety profile, mecapegfilgrastim of two doses groups were all well-tolerated. Fixed $6 \mathrm{mg}$ dose of mecapegfilgrastim exhibited comparable efficacy and safety in comparison with $100 \mu \mathrm{g} / \mathrm{kg}$ during 4 cycles.

Conclusions: Long-acting mecapegfilgrastim $(100 \mu \mathrm{g} / \mathrm{kg}$ and fixed $6 \mathrm{mg})$ is very effective and well tolerated when administered in the primary prophylaxis of chemotherapy induced neutropenia and in 
consecutive-cycle treatment. In some clinical parameters, mecafilgrastim is non-inferior and even superior to filgrastim. The fixed $6 \mathrm{mg}$-dose regimen showed similar efficacy and safety profile compared with $100 \mu \mathrm{g} / \mathrm{kg}$ regimen, and would be the preference in clinical practice, due to the convenient once-per-cycle administration and high-degree treatment compliance for the patients. This study provided new evidence for the novel long-acting rhG-CSF, mecapegfilgrastim, which would be a new alternative for clinical practice for prophylaxis of chemotherapy induced neutropenia.

Keywords: Breast cancer; long-acting granulocyte colony-stimulating factor (long-acting G-CSF); mecapegfilgrastim; filgrastim

Submitted Jul 03, 2019. Accepted for publication Jul 23, 2019.

doi: 10.21037/atm.2019.07.95

View this article at: http://dx.doi.org/10.21037/atm.2019.07.95

\section{Introduction}

Although the advances in targeted therapy and immune therapy, chemotherapy still plays a critical role in the cancer treatment strategy. Meanwhile, chemotherapy induced toxicities can adversely affect the patients' tolerance to chemotherapy and limit the effectiveness of chemotherapy. Neutropenia is a common complication, study shows that $65.5 \%$ of patients experienced a proven hematological toxicity with grade 3-4 neutropenia when treated with docetaxel, doxorubicin and cyclophosphamide (TAC) treatment regimen (1). Moreover, febrile neutropenia (FN) can be life-threatening, which associated with high risk of mortality.

The recombinant human granulocyte colony-stimulating factor (rhG-CSF), filgrastim and pegfilgrastim have been widely used for the prevention of chemotherapy-induced neutropenia (2-5). Filgrastim possesses rapid renal clearance and requires daily administration during chemotherapy. Pegfilgrastim, as the long-acting rhG-CSF, has comparable efficacy and safety profile to filgrastim, but its longer half-life allows once-per-cycle administration during chemotherapy $(6,7)$. Therefore, pegfilgrastim could offer great convenience, which could enable better patient compliance and improved clinical outcomes $(8,9)$. But in China, the US- and EUapproved pegfilgrastim (Neulasta) is not available, leaving the short-acting G-CSF as the major treatment option.

Mecapegfilgramtim (code name HHPG-19K), a longacting rhG-CSF, has been developed by covalently bonding a $19-\mathrm{kDa}$ polyethylene glycol (PEG) to the $\mathrm{N}$ terminus of filgrastim. The previous phase II study has shown that mecapegfilgrastim was well tolerated in non-small cell lung carcinoma (NSCLC) patients receiving platinum-based chemotherapy, the dose of $100 \mu \mathrm{g} / \mathrm{kg}$ was recommended for efficacy evaluating, and the mean plasma half-life value was
55.99 hours (10). The following phase III study showed that once-per-cycle injection of mecapegfilgrastim was as effective and safe as daily filgrastim for prophylaxis of chemotherapyinduced neutropenia in NSCLC patients (11).

For clinically evaluating all G-CSF medicines, patients with (neo)adjuvant breast cancer represent a sensitive population (12). It provides a homogenous patient population which means that they exhibit less inter-patient variation in terms of potential for treatment related toxicity and other confounding factors. Multiple randomized clinical studies have been conducted to demonstrate equivalence between biosimilar and reference G-CSF in breast cancer. In a phase II trial, mecapegfilgrastim preliminarily presented better clinical efficacy as the secondary prophylactic therapy for neutropenia and equal tolerance compared with G-CSF in one cycle treatment in breast cancer patients, and a dose of $100 \mu \mathrm{g} / \mathrm{kg}$ was recommended for further study (13).

In this study, we performed a randomized phase III study in patients with breast cancer. The primary objective was to further evaluate the non-inferiority of mecapegfilgrastim compared with filgrastim as the primary prophylactic therapy during the first cycle of chemotherapy with respect to duration of severe neutropenia (DSN), and also to demonstrate whether the fixed $6 \mathrm{mg}$ dose showed a similar safety and efficacy to the weight-based dose of $100 \mu \mathrm{g} / \mathrm{kg}$. This trial was approved by the National Medical Products Administration of China (registration number: 2010L00501) and registered on ClinicalTrials.gov (NCT01611051).

\section{Methods}

\section{Patients}

The patients who met the following criteria were enrolled: 
pathologically confirmed and previously untreated breast cancer who were eligible to receive neoadjuvant or adjuvant chemotherapy defined by the study protocol; age ranging from 18 to 70 years old; body weight $\geq 45 \mathrm{~kg}$; Eastern Cooperative Oncology Group (ECOG) performance status $0-1$; expected tolerance of chemotherapy $\geq 4$ cycles; adequate organ function: (I) normal bone marrow hematopoietic function without bleeding tendency [international normalized ratio (INR) $<1.5$ ]; (II) adequate hematologic function: hemoglobin $\geq 90 \mathrm{~g} / \mathrm{L}$, white blood cell $(\mathrm{WBC}) \geq 4.0 \times 10^{9} / \mathrm{L}$, absolute neutrophil count (ANC) $\geq 2.0 \times 10^{9} / \mathrm{L}$, platelet count $(\mathrm{PLT}) \geq 100 \times 10^{9} / \mathrm{L}$; (III) adequate renal and hepatic function; (IV) no cardiopulmonary dysfunction; negative pregnancy test (blood sample or urine sample) within 7 days prior to enrollment for child bearing age females who are willing to use reliable contraception methods during the study.

The exclusion criteria include: had a history of bone marrow or stem-cell transplantation; had acute or active infection and received systemic antibiotics within 72 hours before chemotherapy; had hematologic disease could affect bone marrow function; underwent pregnancy or breast feeding; had been enrolled into other clinical trials within 4 weeks before randomization into this study; had previously received pegfilgrastim treatment; hypersensitive to PEG-rhG-CSF or rhG-CSF or other biological agents; had previously received systemic chemotherapy, definitive radiotherapy, palliative radiotherapy within 4 weeks; some special cases that the researchers determined not eligible for the study.

\section{Study design}

This was a randomized, open-label, active-control, multicenter study. The eligible patients received either anthracyclinestaxane (AT) chemotherapy (epirubicin $75 \mathrm{mg} / \mathrm{m}^{2}$ combined with docetaxel $75 \mathrm{mg} / \mathrm{m}^{2}$ ) or adriamycin and cyclophosphamide (AC) chemotherapy (epirubicin $100 \mathrm{mg} / \mathrm{m}^{2}$ combined with cyclophosphamide $600 \mathrm{mg} / \mathrm{m}^{2}$ ) on day 1 of each cycle and every 3 weeks for up to 4 cycles, except for disease progression or unacceptable toxicity.

For cycle 1 , the patients were randomized at a ratio of 1:1:1 to receive a single dose of mecapegfilgrastim $100 \mu \mathrm{g} / \mathrm{kg}$ or a $6 \mathrm{mg}$ fixed dose on day 3 ( $\geq 48$ hours after chemotherapy), or filgrastim $5 \mu \mathrm{g} / \mathrm{kg} /$ day since day 3 ( $\geq 48$ hours after chemotherapy), continuing until a documented ANC $\geq 5.0 \times 10^{9} / \mathrm{L}$ twice or $\mathrm{ANC} \geq 15 \times 10^{9} / \mathrm{L}$ once after the expected nadir, or for up to 14 days, whichever occurred first.
For cycles $2-4$, the patients in mecapegfilgrastim groups continued to receive mecapegfilgrastim $100 \mu \mathrm{g} / \mathrm{kg}$ or a $6 \mathrm{mg}$ fixed dose on day 3 in each cycle. Patients in the control group only received filgrastim treatment in cycle 1 .

Mecapegfilgrastim was provided by Hengrui Medicine Co., Ltd. (Lianyungang, China) and short-acting filgrastim was provided by Kyowa Hakko Kirin China Pharmaceutical Co., Ltd. (Shanghai, China).

\section{Blood monitoring}

In cycle 1 , blood samples were collected within $24 \mathrm{~h}$ of the initiation of chemotherapy and on day $1,3,5,7,8,9,10,11$, $13,15,17$ and 21 during cycle 1 , or until an ANC $\geq 2.0 \times 10^{9}$ / $\mathrm{L}$ was reached.

In cycles 2 to 4, blood samples were taken on day 5, 7, 9, 11,13 and 21 of each cycle. ANC assessments during cycles 2 to 4 were performed within $24 \mathrm{~h}$ of chemotherapy, on day $5,7,9,11,13$ and 21 of each cycle, until an ANC $\geq 2.0 \times 10^{9} / \mathrm{L}$ was achieved.

\section{Endpoints}

The primary endpoint was the mean duration of grade $\geq 3$ neutropenia (defined as ANC $<1.0 \times 10^{9} / \mathrm{L}$ ) during cycle 1 of chemotherapy. The secondary endpoints included the incidence of grade $\geq 3$ and grade 4 neutropenia in cycles $1-4$, the mean duration of grade $\geq 3$ neutropenia in cycles $2-4$, the mean duration of grade 4 neutropenia in cycles $1-4$, the incidence of $\mathrm{FN}$ (defined as body temperature $\geq 38.5^{\circ} \mathrm{C}$ concurrent with ANC $<1.0 \times 10^{9} / \mathrm{L}$ ) in cycles $1-4$.

The safety assessment was measured by reports of adverse events (AEs), changes in clinical laboratory values, vital signs and physical examinations.

\section{Statistical analysis}

This study was designed to show each of the mecapegfilgrastim arms is non-inferior to the filgrastim arm. The primary efficacy analyses were performed in the full analysis set (FAS). For duration of grade 3 or higher ANC decreases during cycle 1, we hypothesized the noninferior margin as 1 day. Using an analysis of covariance (ANCOVA) model, the difference between patients treated with mecapegfilgrastim and patients treated with filgrastim would be calculated together with $95 \%$ confidence interval (CI). Non-inferiority would be established if the upper limit of the $95 \%$ CI was less than 1 day. If non-inferiority was 


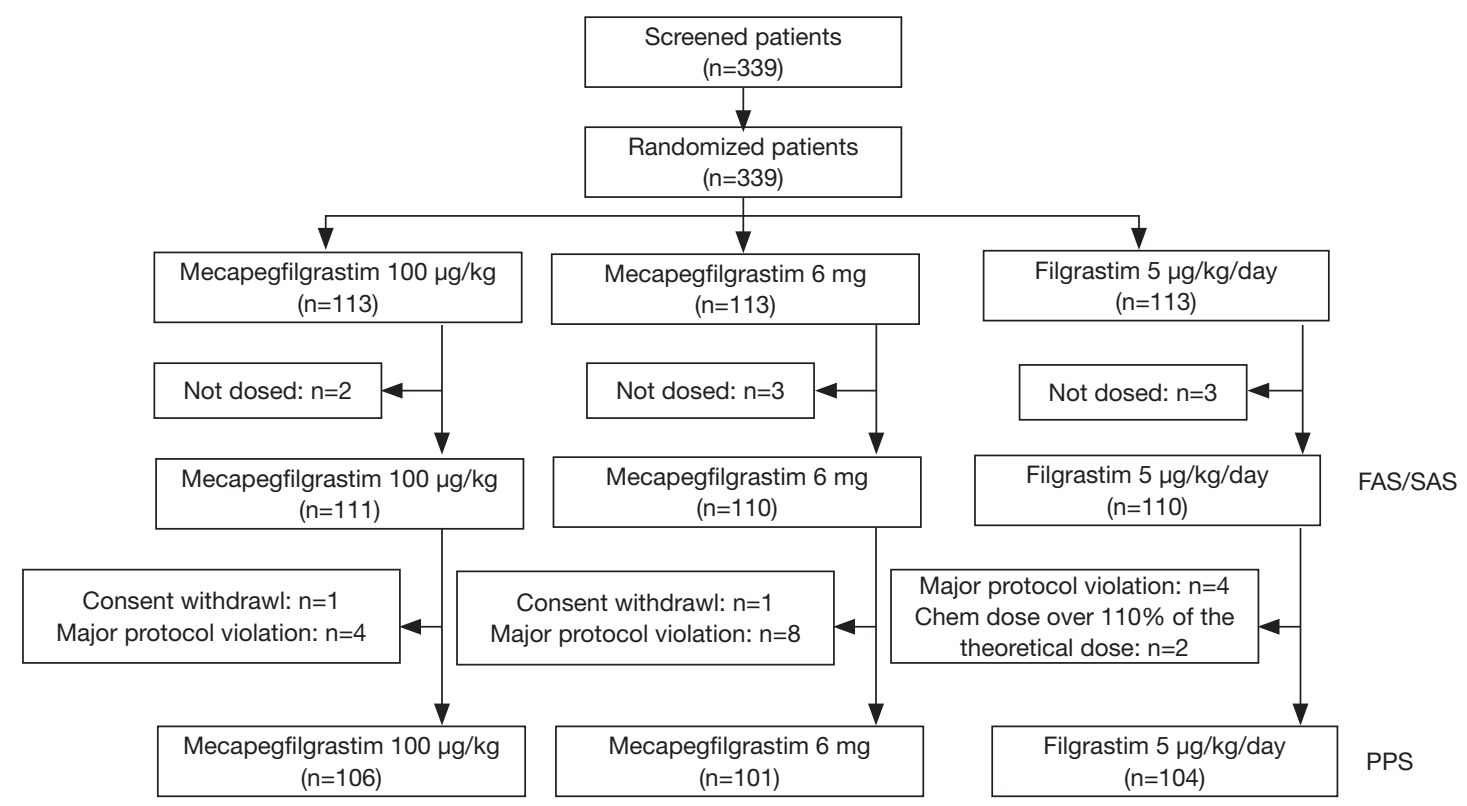

Figure 1 Patient disposition in the study. FAS, full analysis set; SAS, safety analysis set; PPS, per protocol set.

established, the upper limit of the $95 \%$ CI could be further compared to 0 for assessment of superiority.

The sample size was calculated based on the primary end point of the duration of grade $\geq 3$ ANC decrease at the first cycle of chemotherapy. Assuming a pre-specified non-inferiority margin of 1 day and a common standard deviation of 2 days, it was calculated that a total of 258 patients (86 per arm) were required to assess non-inferiority of mecapegfilgrastim (100 $\mu \mathrm{g} / \mathrm{kg}$ or $6 \mathrm{mg})$ to the filgrastim at a one-sided significance level of $2.5 \%$ with $90 \%$ power. In order to allow for $20 \%$ of drop-outs and major protocol violations, a total of 330 patients were planned to be randomized with 110 patients in each arm. All statistical analysis was conducted using SAS 9.3 software.

\section{Results}

\section{Baseline characteristics}

From March 2012 to November 2012, total of 339 patients from 22 centers in China were recruited into the study (Table S1). During the study, eight patients were excluded from the FAS, because they did not receive the treatment after randomization. At the end of cycle 1, total 331 (97.64\%) patients received at least one dose of study drug and were included into the FAS. The per protocol set (PPS) included 311 patients, excluding 2 consent withdrawl, 16 major protocol violation, and 2 overdose chemotherapy treatment. The number of patients eligible for safety analysis set (SAS) was 331. Figure 1 illustrated the detailed patient disposition in each group in this study.

Demographic characteristics and disease status of patients were similar across treatment groups. Baseline vital signs, physical examination and general clinical characteristics were comparable among the three groups. Baseline ANC levels were within the normal range for all three groups and there were no statistically significant differences among the three groups at baseline (Table 1).

\section{Primary efficacy endpoint}

The efficacy analysis of FAS and PPS led to identical conclusions, only the results of FAS were reported here.

\section{The mean duration of grade $\geq 3$ neutropenia}

In cycle 1 , the adjusted mean duration of grade $\geq 3$ neutropenia was 1.06 (95\% CI: $0.65,1.26)$ days in mecapegfilgrastim $100 \mu \mathrm{g} / \mathrm{kg}$ group, 1.23 (95\% CI: 0.84, 1.88) days in mecapegfilgrastim $6 \mathrm{mg}$ group, and 2.06 (95\% CI: 1.66, 2.46) days in the filgrastim group (Figure 2).

The mean difference between mecapegfilgrastim $100 \mu \mathrm{g} / \mathrm{kg}$ and filgrastim was -1.00 (95\% CI: -1.52 , $-0.48)$, the mean difference between mecapegfilgrastim $6 \mathrm{mg}$ and filgrastim was -0.83 (95\% CI: $-1.36,-0.30$ ). (Figure 2). The upper bounds of $95 \%$ CI for the mean 
Table 1 Demographic and clinical characteristics for full analysis population

\begin{tabular}{|c|c|c|c|}
\hline Characteristic & Mecapegfilgrastim $100 \mu \mathrm{g} / \mathrm{kg}$ & Mecapegfilgrastim 6 mg & Filgrastim $5 \mu \mathrm{g} / \mathrm{kg} /$ day \\
\hline Female & $111[100]$ & $110[100]$ & $110[100]$ \\
\hline Mean age, years $( \pm S D)$ & $48.21 \pm 8.55$ & $48.03 \pm 9.01$ & $47.37 \pm 8.60$ \\
\hline Mean weight, kg ( \pm SD) & $59.54 \pm 8.55$ & $59.05 \pm 9.60$ & $60.39 \pm 9.16$ \\
\hline \multicolumn{4}{|l|}{ ECOG performance status, $\mathrm{n}(\%)$} \\
\hline 0 & $72(64.86)$ & $72(65.45)$ & 69 (62.73) \\
\hline 1 & $39(35.14)$ & $38(34.55)$ & $41(37.27)$ \\
\hline \multicolumn{4}{|l|}{ Regimen, n (\%) } \\
\hline
\end{tabular}

SD, standard deviation; BSA, body surface area; ECOG, Eastern Cooperative Oncology Group; AC, adriamycin and cyclophosphamide; AT, anthracyclines-taxane; ANC, absolute neutrophil count.

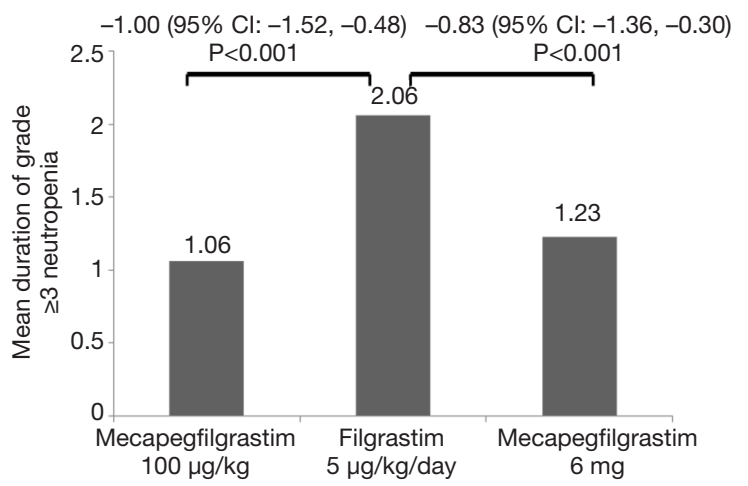

Figure 2 The mean duration of grade $\geq 3$ neutropenia in first treatment cycle.

difference between mecapegfilgrastim and filgrastim were all $<1$ day (the predefined non-inferiority margin), the study met its primary endpoint.

\section{Secondary endpoints}

The incidence of grade $\geq 3$ and grade 4 neutropenia in cycles 1-4

In cycle 1 , there are 56 patients $(50.45 \%)$ in mecapegfilgrastim $100 \mu \mathrm{g} / \mathrm{kg}$ arm, 56 patients (50.91\%) in mecapegfilgrastim $6 \mathrm{mg}$ arm, and 73 patients $(66.36 \%)$ in filgrastim arm experienced grade $\geq 3$ neutropenia. Compared with filgrastim arm, the incidence of grade $\geq 3$ neutropenia was significantly lower in patient treated with mecapegfilgrastim $100 \mu \mathrm{g} / \mathrm{kg}(\mathrm{P}=0.0147)$ and mecapegfilgrastim $6 \mathrm{mg}$ $(\mathrm{P}=0.0064)$. There was no difference between the two mecapegfilgrastim arms in the incidence of grade $\geq 3$ neutropenia $(\mathrm{P}=0.9470)$. There are 37 patients $(33.33 \%)$ in mecapegfilgrastim $100 \mu \mathrm{g} / \mathrm{kg}$ arm, 33 patients (30.00\%) in mecapegfilgrastim $6 \mathrm{mg}$ arm, and 51 patients (46.36\%) in filgrastim arm experienced grade 4 neutropenia. Compared with filgrastim, the incidence of grade 4 neutropenia was significantly lower in patient treated with mecapegfilgrastim $100 \mu \mathrm{g} / \mathrm{kg}(\mathrm{P}=0.0454)$ and mecapegfilgrastim $6 \mathrm{mg}$ $(\mathrm{P}=0.0036)$. There was no difference between the two mecapegfilgrastim arms in the incidence of grade 4 neutropenia $(\mathrm{P}=0.5688)$ (Table 2$)$.

In cycle 2, 13 patients $(15.66 \%)$ in mecapegfilgrastim $100 \mu \mathrm{g} / \mathrm{kg}$ arm and 18 patients (21.18\%) in mecapegfilgrastim $6 \mathrm{mg}$ arm experienced grade $\geq 3$ neutropenia, there was no difference between these two groups $(\mathrm{P}=0.6917)$. There are seven patients (8.43\%) in mecapegfilgrastim $100 \mu \mathrm{g} / \mathrm{kg}$ arm and nine patients (10.59\%) in mecapegfilgrastim $6 \mathrm{mg}$ arm experienced grade 4 neutropenia, there was no difference between these two groups $(\mathrm{P}=0.9469)$.

In cycle 3 , the incidences of grade $\geq 3$ neutropenia 
Table 2 Secondary endpoints results in cycle 1

\begin{tabular}{|c|c|c|c|c|}
\hline Secondary endpoints & \multicolumn{2}{|c|}{ Mecapegfilgrastim } & Filgrastim & $P$ value \\
\hline Incidence of grade $\geq 3$ neutropenia, $n(\%)$ & $56(50.45)^{\star}$ & $56(50.91)^{\star}$ & $73(66.36)$ & 0.0129 \\
\hline Incidence of grade 4 neutropenia, n (\%) & $37(33.33)^{\star}$ & $33(30.00)^{*}$ & $51(46.36)$ & 0.0090 \\
\hline Duration of grade 4 neutropenia, (days, mean \pm SD) & $0.61 \pm 0.96^{*}$ & $0.54 \pm 0.88^{*}$ & $1.02 \pm 1.24$ & 0.0003 \\
\hline
\end{tabular}

*, $\mathrm{P}<0.05$ versus filgrastim arm. SD, standard deviation; FN, febrile neutropenia.

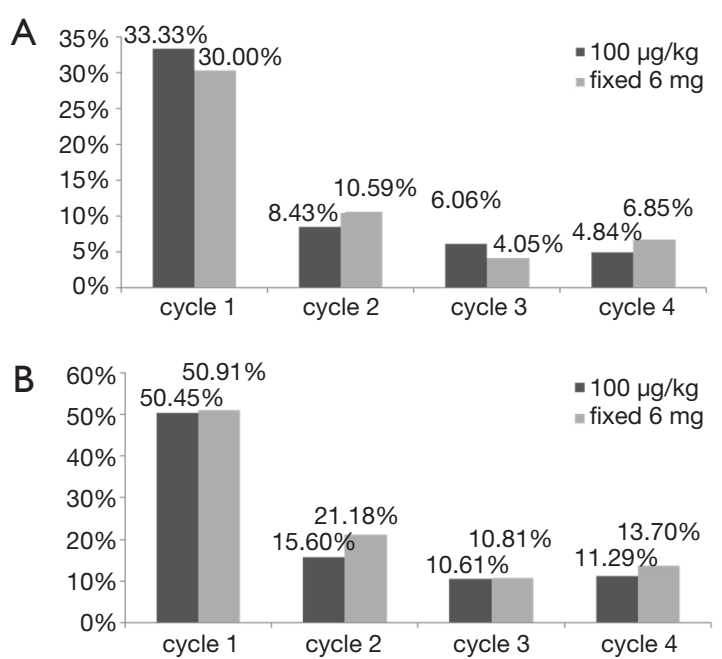

Figure 3 The incidence of neutropenia in mecapegfilgrastim $100 \mu \mathrm{g} / \mathrm{kg}$ group and fixed $6 \mathrm{mg}$ group. (A) The incidence of grade 4 neutropenia; (B) the incidence of grade $\geq 3$ neutropenia.

were $10.61 \%$ and $10.81 \%$ in mecapegfilgrastim $100 \mu \mathrm{g} / \mathrm{kg}$ and $6 \mathrm{mg}$ groups. The incidences of grade 4 neutropenia were respectively $6.06 \%$ and $4.05 \%$ in these two groups. No difference was found between the two groups.

In cycle 4 , the incidence of grade $\geq 3$ neutropenia was $11.29 \%$ and $13.70 \%$ in mecapegfilgrastim $100 \mu \mathrm{g} / \mathrm{kg}$ and $6 \mathrm{mg}$ groups. The incidence of grade 4 neutropenia was respectively $4.84 \%$ and $6.85 \%$ in these two groups. No difference was found between the two groups.

It was noted that there was a decreased trend in the incidence of grade $\geq 3$ and grade 4 neutropenia as the treatment cycle increased (Figure 3).

The mean duration of grade $\geq 3$ neutropenia in cycles $2-4$ In cycle 2 , the mean duration of grade $\geq 3$ neutropenia was $0.36 \pm 0.99$ days and $0.44 \pm 0.92$ days in mecapegfilgrastim
$100 \mu \mathrm{g} / \mathrm{kg}$ and $6 \mathrm{mg}$ groups. In cycle 3, the mean duration of grade $\geq 3$ neutropenia was $0.23 \pm 0.72$ days and $0.26 \pm 0.79$ days in mecapegfilgrastim $100 \mu \mathrm{g} / \mathrm{kg}$ and $6 \mathrm{mg}$ groups. In cycle 4 , the mean duration of grade $\geq 3$ neutropenia was $0.63 \pm$ 2.39 days and $1.04 \pm 3.14$ days in the two groups. No difference was found between the two groups.

The mean duration of grade 4 neutropenia in cycles 1-4 In cycle 1 , the mean duration of grade 4 neutropenia was $0.61 \pm 0.96,0.54 \pm 0.88$, and $1.02 \pm 1.24$ days in mecapegfilgrastim $100 \mu \mathrm{g} / \mathrm{kg}$ group, mecapegfilgrastim $6 \mathrm{mg}$ group, and filgrastim group. The mean difference between mecapegfilgrastim $100 \mu \mathrm{g} / \mathrm{kg}$ and filgrastim groups was -0.40 (95\% CI: $-0.73,-0.08)$, the mean difference between mecapegfilgrastim $6 \mathrm{mg}$ and filgrastim groups was -0.51 (95\% CI: $-0.84,-0.18$ ), all the differences were statistically significant. The mean difference between two mecapegfilgrastim groups was 0.10 (95\% CI: $-0.22,0.43)$ which was not statistically significant (Table 2).

In cycle 2, the mean duration of grade 4 neutropenia was $0.16 \pm 0.57$ and $0.22 \pm 0.70$ days in mecapegfilgrastim $100 \mu \mathrm{g} / \mathrm{kg}$ and $6 \mathrm{mg}$ groups. In cycle 3, the mean duration of grade 4 neutropenia was $0.12 \pm 0.48$ and $0.08 \pm 0.40$ days. In cycle 4 , the mean duration of grade 4 neutropenia was $0.08 \pm 0.38$ and $0.53 \pm 2.35$ days. All the differences between the two groups were not significant.

\section{The incidence of $\mathrm{FN}$}

In cycle 1 , there are $5(4.50 \%), 0(0 \%)$, and $2(1.82 \%)$ patients experienced $\mathrm{FN}$ in mecapegfilgrastim $100 \mu \mathrm{g} / \mathrm{kg}$ group, mecapegfilgrastim $6 \mathrm{mg}$ group and filgrastim group. There was no significant difference between the three groups (Table 2). During cycles 2 to 4, no FN was developed in patients in mecapegfilgrastim $100 \mu \mathrm{g} / \mathrm{kg}$ and mecapegfilgrastim $6 \mathrm{mg}$ groups. 
Table 3 Subgroup analysis of duration of grade $\geq 3$ neutropenia in cycle 1

\begin{tabular}{|c|c|c|c|c|}
\hline Subgroup & \multicolumn{2}{|c|}{ Mecapegfilgrastim } & Filgrastim & $P$ value \\
\hline \multicolumn{5}{|c|}{ Body weight days, mean $\pm \mathrm{SD}(\mathrm{N})$} \\
\hline$\leq 70 \mathrm{~kg}$ & $1.17 \pm 1.28[102]^{*}$ & $1.32 \pm 1.82[95]^{\star}$ & $2.20 \pm 2.17[94]$ & $<0.0001$ \\
\hline$>70 \mathrm{~kg}$ & $0.44 \pm 0.73[9]$ & $1.40 \pm 2.29[15]$ & $1.69 \pm 1.62[16]$ & 0.2248 \\
\hline$A C$ & $1.32 \pm 1.46[50]$ & $1.59 \pm 2.47[49]$ & $2.06 \pm 2.18[50]$ & 0.0886 \\
\hline AT & $0.93 \pm 1.05[61]^{\star}$ & $1.11 \pm 1.20[61]^{\star}$ & $2.18 \pm 2.05[60]$ & $<0.0001$ \\
\hline
\end{tabular}

*, $\mathrm{P}<0.05$ versus filgrastim arm. SD, standard deviation; AC, adriamycin and cyclophosphamide; AT, anthracyclines-taxane.

\section{Subgroup analysis of primary endpoints}

In order to determine whether the stratification factors (age, chemotherapy regimens) confounded the assessment of efficacy, an exploratory subgroup analyses according to the baseline body weight ( $\leq 70 v s .>70 \mathrm{~kg}$ ) and chemotherapy (AC $v s$. AT) was performed for cycle 1 .

For patients with body weight $\leq 70 \mathrm{~kg}$, the mean duration of grade $\geq 3$ neutropenia were respectively $1.17 \pm 1.28,1.32 \pm 1.82$ and $2.20 \pm 2.17$ days, compared with filgrastim group, the mean duration of grade $\geq 3$ neutropenia were significantly shorter in patients with mecapegfilgrastim $100 \mu \mathrm{g} / \mathrm{kg}$ and $6 \mathrm{mg}$ treatment, the difference were respectively -0.98 (95\% CI: $-1.56,-0.39$ ) and -0.91 (95\% CI: $-1.51,-0.31)$. There is no difference between two mecapegfilgrastim arms. For patients with body weight $>70 \mathrm{~kg}$, no difference was found in the mean duration of grade $\geq 3$ neutropenia between the three groups (Table 3).

For patients treated with AT chemotherapy, the mean duration of grade $\geq 3$ neutropenia respectively $0.93 \pm 1.05$, $1.11 \pm 1.20$ and $2.18 \pm 2.05$ days, compared with filgrastim group, the mean duration of grade $\geq 3$ neutropenia were significantly shorter in patients with mecapegfilgrastim two dose regimens treatment, the difference were respectively -1.20 (95\% CI: $-1.81,-0.59)$ and -1.10 (95\% CI: -1.71 , -0.49). For patients treated with AC chemotherapy, there was no significant difference between the three groups (Table 3).

\section{Safety analysis}

In cycle 1, total 320 patients experienced AEs in mecapegfilgrastim $100 \mu \mathrm{g} / \mathrm{kg}$ arm (105 patients, 94.59\%), mecapegfilgrastim $6 \mathrm{mg}$ arm (107 patients, 97.27\%) and filgrastim arm (108 patients, 98.18\%). Total seven patients reported serious adverse events (SAEs) in mecapegfilgrastim $100 \mu \mathrm{g} / \mathrm{kg}$ (three patients), mecapegfilgrastim $6 \mathrm{mg}$ (one patient) and filgrastim (three patients) arms. All of the SAEs were considered not related to investigational treatment by the investigators. No unexpected AE was observed. The most frequently reported AEs possibly related to treatment were shown in Table 4. Grade 3 AEs were reported in mecapegfilgrastim $100 \mu \mathrm{g} / \mathrm{kg}$ group (two patients), $6 \mathrm{mg}$ fixed dose group (three patients) and filgrastim group (two patients). Overall, no significant difference was detected among the three groups in terms of the incidence of all AEs, the treatment related AEs, and the SAEs.

\section{Discussion}

Neutropenia is the main dose-limiting toxicity of chemotherapy, always leading to dosage adjustment or treatment interruption, which could compromise the efficacy, so it is very important to ensure sufficient treatment cycles and dosage for chemotherapy. In a phase II trial, mecapegfilgrastim demonstrated better clinical efficacy and similar safety profile as the secondary prophylactic therapy for neutropenia compared with filgrastim in breast cancer patients (13).

However, in above phase II trial, the evaluation of the efficacy and safety of mecapegfilgrastim was mainly focused on the secondary prophylactic therapy in one treatment cycle. This phase III trial was designed to further investigate the efficacy and safety of mecapegfilgrastim as the primary prophylactic therapy in four consecutive treatment cycles, and also to explore whether the fixed $6 \mathrm{mg}$ dosage showed a similar safety and efficacy to the weight-based dose of 
Table 4 The most frequently reported AEs related to treatment drugs

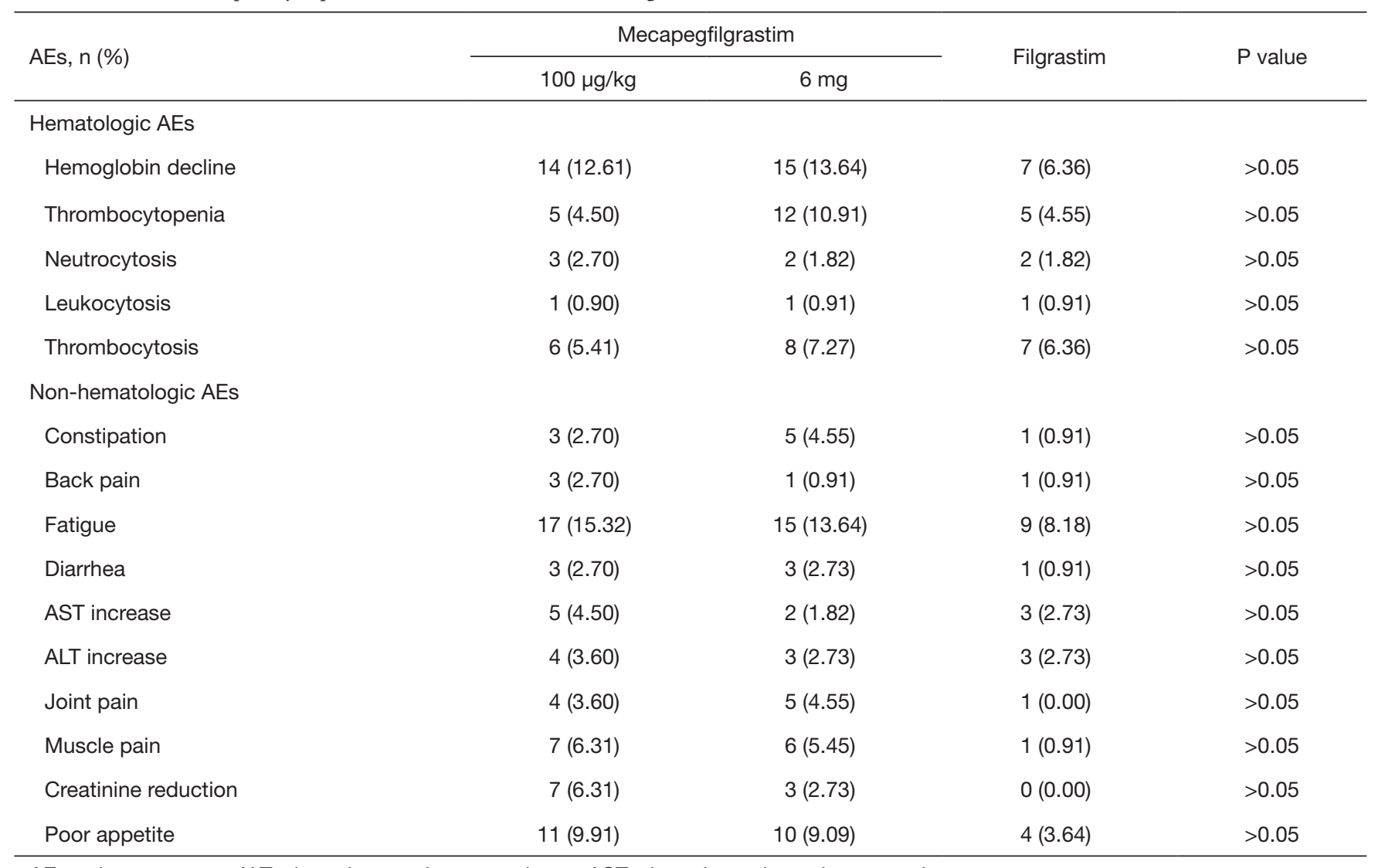

AEs, adverse events; ALT, glutamic-pyruvic transaminase; AST, glutamic-oxaloacetic transaminase.

$100 \mu \mathrm{g} / \mathrm{kg}$. The results of this study showed that the single dose of mecapegfilgrastim (at either $100 \mu \mathrm{g} / \mathrm{kg}$ or $6 \mathrm{mg}$ ) was as safe and effective as daily injections of filgrastim as the primary prophylactic therapy in the first chemotherapy cycle in breast cancer patients, which was consistent with the results of some other phase II and phase III studies in breast cancer or other malignant tumors.

DSN was considered as a sensitive endpoint in assessing biosimilarity of filgrastim in (neo) adjuvant breast cancer, any variations in DSN can be considered as a direct consequence of differences between activity of reference and biosimilar filgrastim (12). Moreover, risk of infection is directly proportional to severity and duration of neutropenia, making DSN a clinically relevant endpoint $(14,15)$. As for the primary endpoint of this study, the mean duration of grade $\geq 3$ neutropenia in both mecapegfilgrastim groups were non-inferior to that in the filgrastim group in cycle one. The mean difference of the duration of grade $\geq 3$ neutropenia between mecapegfilgrastim $100 \mu \mathrm{g} / \mathrm{kg}$ and filgrastim was -1.00 day ( $95 \%$ CI: $-1.52,-0.48$ day), the difference between mecapegfilgrastim $6 \mathrm{mg}$ and filgrastim was -0.83 day (95\% CI: $-1.36,-0.30$ day). The upper bounds of $95 \%$ CI for the mean difference between mecapegfilgrastim and filgrastim were all within the predefined margin of 1 day, which indicated that the efficacy of mecapegfilgrastim in reducing duration of grade $\geq 3$ neutropenia was non-inferior to filgrastim.

Furthermore, the upper bounds of $95 \%$ CI for the mean difference between mecapegfilgrastim and filgrastim were all $<0$, which indicated that compared with filgrastim, mecapegfilgrastim at dosage of either $100 \mu \mathrm{g} / \mathrm{kg}$ or $6 \mathrm{mg}$ might be superior in reducing duration of grade $\geq 3$ neutropenia. The superiority might be the result of the longer half-life time of 55.99 hours and the unique linking structure of mecapegfilgrastim. For the duration of grade 4 neutropenia, compared with filgrastim, the duration of grade 4 neutropenia were significantly shorter in patients treated with mecapegfilgrastim $100 \mu \mathrm{g} / \mathrm{kg}$ and the fixed dosage of $6 \mathrm{mg}$. The reduced DSN could be associated with decreased risk of infection and shorter period of 
hospitalization, which could save and make good use of the limited medical resources to serve more patients, and make the patients spend more time with their family at home.

In this study, for some secondary endpoints (the incidence of grade $\geq 3$ and grade 4 neutropenia, the mean duration of grade 4 neutropenia), there were significant differences between two dosage groups of mecapegfilgrastim and filgrastim, indicating that in some parameters, mecapegfilgrastim may be better than filgrastim in supporting cytotoxic chemotherapy. These results were consistent with the results from phase III study of the mecapegfilgrastim in NSCLC, which showed that for the duration of grade 4 neutropenia in cycles 2 to 4 and for the incidence of $\mathrm{FN}$, the differences between mecapegfilgrastim and filgrastim were significant (11). And the phase II trial of mecapegfilgrastim in breast cancer also showed similar results (13). A systematic review also revealed similar results, showing better efficacy and effectiveness for pegfilgrastim than filgrastim (16). All these results suggest that longeracting rhG-CSF might provide additional clinical benefit, the mechanism for such findings was unclear.

In our study, we further evaluated the efficacy of mecapegfilgrastim in consecutive-cycle applications, which showed a declining trend of the incidence of grade $\geq 3$ and grade 4 neutropenia along with the treatment cycles increased (Figure 2), which indicated that patients may benefit more from consecutive-cycle treatment of mecapegfilgrastim (at either $100 \mu \mathrm{g} / \mathrm{kg}$ or $6 \mathrm{mg}$ ) in prevention of severe neutropenia. This hypothesis would be further evaluated in the well-designed clinical trials.

In clinical practice, a fixed-dose regimen would begenerally preferred for administration. However, there were concerns about the fixed dosage for the lack of efficacy in over-weighted patients and occurrence of sever AEs in less-weighted patients. So, in this study, we added a fixeddose group of mecapegfilgrastim at $6 \mathrm{mg}$, and compared the efficacy of the two dosage regimens of mecapegfilgrastim $(100 \mu \mathrm{g} / \mathrm{kg}$ and a fixed dosage of $6 \mathrm{mg})$ in different-weight subgroups in all 4 cycles. The results showed, there was no significant difference between the fixed $6 \mathrm{mg}$ and $100 \mu \mathrm{g} / \mathrm{kg}$ of mecapegfilgrastim in duration of grade $\geq 3$ neutropenia, incidence of grade $\geq 3$ neutropenia and incidence of bone pain during all cycles. The previous studies also demonstrated that mecapegfilgrastim fixed $6 \mathrm{mg}$ or $100 \mu \mathrm{g} / \mathrm{kg}$ dosage provided comparable benefit as filgrastim $(2,11)$. Thus, the efficacy and safety of the fixed $6 \mathrm{mg}$-dose regimen is appropriate for patients, and should be recommended in the clinical practice in terms of the convenience of administration.
In China, AT and AC chemotherapy regimens were commonly used in clinical practice, and were also recommended in Chinese treatment guidelines for breast cancer patients. These two regimens have proven dose limiting hematological toxicity with grade 3-4 neutropenia. In this study, for AT regimen, patients treated with mecapegfilgrastim experienced shorter duration of grade $\geq 3$ neutropenia compared with filgrastim. For patients treated with AC regimen, mecapegfilgrastim exhibited comparable efficacy with filgrastim for the duration of grade $\geq 3$ neutropenia. These results indicated that patients treated with mecapegfilgrastim might benefit more for neutropenia prophylaxis when they treated with AT chemotherapy, which possessed stronger myelosuppression than AC chemotherapy.

For safety profile, there was no significant difference between mecapegfilgrastim (either $100 \mu \mathrm{g} / \mathrm{kg}$ or fixed $6 \mathrm{mg}$ ) and filgrastim in terms of the incidence of all AEs, including the common events of pain and decreased hemoglobin. In this phase III study, no unexpected AEs, fixed-dosage related AEs, nor consecutive-cycle related AEs were found. All of the SAEs were considered not related to the investigational treatment by the investigators. Patients in megapegfilgrastim groups were well tolerated.

There is also a limitation in this study. We only included the breast cancer patients in AT/AC chemotherapy regimens limited by the rules of randomized-control-study design. In the future, we plan to evaluate the effectiveness and safety of mecapegfilgrastim further in the real-world study, in which more practical chemotherapy regimens would be included.

In conclusion, this study demonstrated that longacting mecapegfilgrastim $(100 \mu \mathrm{g} / \mathrm{kg}$ and fixed $6 \mathrm{mg})$ is very effective and well tolerated when administered in the primary prophylaxis of chemotherapy induced neutropenia and in consecutive-cycle treatment. In some clinical parameters, mecafilgrastim is non-inferior and even superior to filgrastim. The fixed $6 \mathrm{mg}$-dose regimen showed similar efficacy and safety profile compared with $100 \mu \mathrm{g} / \mathrm{kg}$ regimen, and would be the preference in clinical practice, due to the convenient once-per-cycle administration and high-degree treatment compliance for the patients. This study provided new evidence for the novel long-acting rhGCSF, mecapegfilgrastim, which would be a new alternative for clinical practice for prophylaxis of chemotherapy induced neutropenia.

\section{Acknowledgments}

The authors acknowledge the contribution of: Huang 
Zhongcheng (Department of General Surgery, Hunan Provincial Hospital, Changde, China); Liu Yunpeng (Department of Oncology, The First Affiliated Hospital, China Medical University, Shenyang, China); Wu Youhua (Department of Oncology, The First Hospital University of South China, Hengyang, China); Li Xinzheng (Department of Breast Surgery, Shanxi Cancer Hospital, Taiyuan, China); Zhang Tao (Department of Oncology, Chengdu Military General Hospital, Chengdu, China); Wang Ningju (Department of Oncology, Affiliated Hospital of Ninxia Medical University, Yinchuan, China); Gao Jianfei (Department of Oncology, Wuhan General Hospital, Guangzhou Command of PLA, Wuhan, China); Sun Heqing (Department of Breast Surgery, Yangzhou First People's Hospital, Yangzhou, China); Huang Feizhou (Department of General Surgery, The Third Xiangya Hospital Of Central-South University, Changsha, China); Dang Chengxue (Department of Tumor Surgery, The First Affiliated Hospital, Medical school of Xi'an Jiaotong University, Xi'an, China); Ding Kefeng (Department of Oncology, The Second Affiliated Hospital, College of Medicine, Zhejiang University, Hangzhou, China); Liu Yunjiang (Department of Surgery, The Fourth Hospital of Hebei Medical University, Shijiazhuang, China) for their contributions to the enrollment of trial subjects. Cunnan Dong assisted with the writing of this manuscript. This study was funded by Hengrui Medicine Co., Ltd. (Lianyungang, China).

\section{Footnote}

Conflicts of Interest: The authors have no conflicts of interest to declare.

Ethics Statement: The authors are accountable for all aspects of the work in ensuring that questions related to the accuracy or integrity of any part of the work are appropriately investigated and resolved. This study was approved by the Ethics Committee of the medical center (2011-11-102), and the protocol was conducted in accordance with the Declaration of Helsinki and Good Clinical Practice guidelines. All patients or their guardians signed the informed consent before enrollment. This trial was approved by the National Medical Products Administration of China (registration number: 2010L00501) and registered on ClinicalTrials.gov (NCT01611051).

\section{References}

1. Martin M, Pienkowski T, Mackey J, et al. Adjuvant docetaxel for node-positive breast cancer. N Engl J Med 2005;352:2302-13.

2. Green MD, Koelbl H, Baselga J, et al. A randomized double-blind multicenter phase III study of fixed-dose single-administration pegfilgrastim versus daily filgrastim in patients receiving myelosuppressive chemotherapy. Ann Oncol 2003;14:29-35.

3. Holmes FA, O'Shaughnessy JA, Vukelja S, et al. Blinded, randomized, multicenter study to evaluate single administration pegfilgrastim once per cycle versus daily filgrastim as an adjunct to chemotherapy in patients with high-risk stage II or stage III/IV breast cancer. J Clin Oncol 2002;20:727-31.

4. Nasti G, Talamini R, Antinori A, et al. AIDS-related Kaposi's sarcoma: evaluation of potential new prognostic factors and assessment of the AIDS clinical trial group staging system in the HAART era-the Italian cooperative group on AIDS and tumors and the Italian cohort of patients naive from antiretrovirals. J Clin Oncol 2003;21:2876-82.

5. Vogel CL, Wojtukiewicz MZ, Carroll RR, et al. First and subsequent cycle use of pegfilgrastim prevents febrile neutropenia in patients with breast cancer: a multicenter, double-blind, placebo-controlled phase III study. J Clin Oncol 2005;23:1178-84.

6. Biganzoli L, Untch M, Skacel T, et al. Neulasta (pegfilgrastim): a once-per-cycle option for the management of chemotherapy-induced neutropenia. Semin Oncol 2004;31:27-34.

7. Molineux G. The design and development of pegfilgrastim (PEG-rmetHuG-CSF, Neulasta). Curr Pharm Des 2004;10:1235-44.

8. Cooper KL, Madan J, Whyte S, et al. Granulocyte colonystimulating factors for febrile neutropenia prophylaxis following chemotherapy: systematic review and metaanalysis. BMC Cancer 2011;11:404.

9. Weycker D, Malin J, Barron R, et al. Comparative effectiveness of filgrastim, pegfilgrastim, and sargramostim as prophylaxis against hospitalization for neutropenic complications in patients with cancer receiving chemotherapy. Am J Clin Oncol 2012;35:267-74.

10. Yan B, Zhang W, Lu F, et al. Safety of polyethylene glycol recombinant human granulocyte colony-stimulating factor in treating non-small cell lung cancer patients at I b stage. 
Asian Pac J Trop Med 2013;6:912-5.

11. Zhou C, Huang Y, Wang D, et al. A randomized multicenter phase III study of single administration of mecapegfilgrastim (HHPG-19K), a pegfilgrastim biosimilar, for prophylaxis of chemotherapy-induced neutropenia in patients with advanced non-small-cell lung cancer (NSCLC). Clin Lung Cancer 2016;17:119-27.

12. Krendyukov A, Schiestl M, Höbel N, et al. Clinical equivalence with G-CSF biosimilars: methodologic approach in a (neo) adjuvant setting in non-metastatic breast cancer. Support Care Cancer 2018;26:33-40.

13. Wang T, Wu B, Hu X, et al. A randomized multicenter phase II trial of mecapegfilgrastim single administration versus granulocyte colony-stimulating growth factor on

Cite this article as: $\mathrm{Xu} \mathrm{F}$, Zhang $\mathrm{Y}$, Miao Z, Zeng $\mathrm{X}$, Wu B, Cai L, Liu J, Wang S, Hu X, Zheng W, Chen Z, Yang Q, Jiang $Z$. Efficacy and safety of mecapegfilgrastim for prophylaxis of chemotherapy-induced neutropenia in patients with breast cancer: a randomized, multicenter, active-controlled phase III trial. Ann Transl Med 2019;7(18):482. doi: 10.21037/ atm.2019.07.95 treating chemotherapy-induced neutropenia in breast cancer patients. Ann Transl Med 2019;7:196.

14. Bodey GP, Buckley M, Sathe YS, et al. Quantitative relationships between circulating leukocytes and infection in patients with acute leukemia. Ann Intern Med 1966;64:328-40.

15. Crawford J, Dale DC, Lyman GH. Chemotherapyinduced neutropenia: risks, consequences, and new directions for its management. Cancer 2004;100:228-37.

16. Pfeil AM, Allcott K, Pettengell R, et al. Efficacy, effectiveness and safety of long-acting granulocyte colonystimulating factors for prophylaxis of chemotherapyinduced neutropenia in patients with cancer: a systematic review. Support Care Cancer 2015;23:525-45. 
Table S1 Medical centers participating in this study

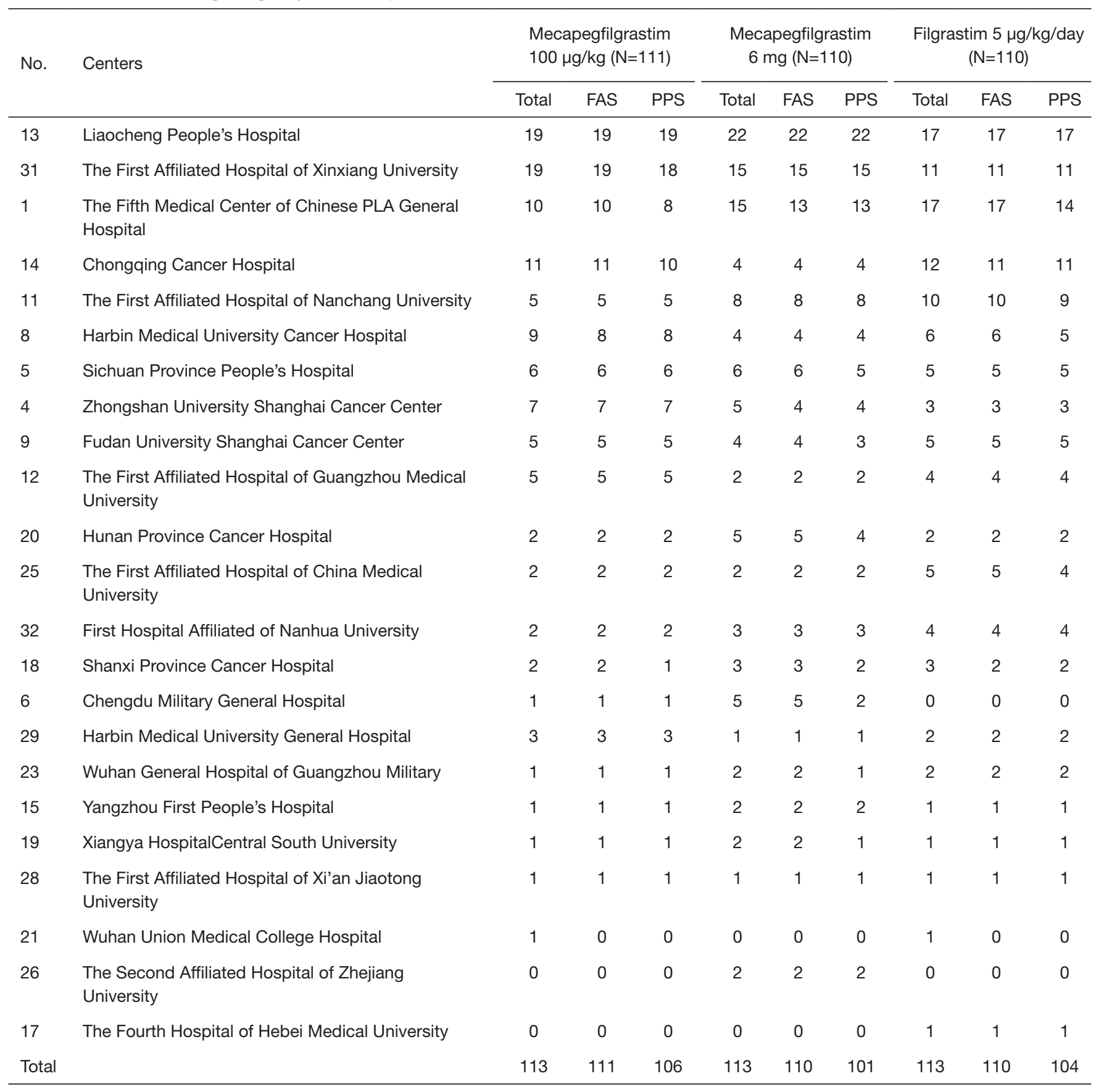

